\title{
Design and Optimization of Radiation Pattern Reconfigurable Nanoloop Antennas
}

\author{
Ryan J. Chaky*, Jogender Nagar, and \\ Douglas H. Werner \\ Department of Electrical Engineering \\ Penn State University \\ University Park, PA, USA \\ rjc74@psu.edu, jun163@psu.edu, and dhw@psu.edu
}

\author{
Arnold F. McKinley \\ Department of Electronic and Electrical Engineering \\ University College London \\ London, UK \\ arni.mckinley@ucl.ac.uk
}

\author{
Mario F. Pantoja \\ Department of Electromagnetics \\ University of Granada \\ Granada, Spain \\ mario@ugr.es
}

\begin{abstract}
The loading of antennas greatly expands the design space by making otherwise challenging performance goals more easily realizable. It is demonstrated that the pairing of the analytical theory of loop antennas with a powerful global optimizer can achieve designs that offer significant radiation pattern shaping in both the RF and optical regimes.
\end{abstract}

Keywords-antennas, nano-antennas, adaptive and reconfigurable antennas, optimization.

Antennas have remained an important component of modern wireless communication infrastructure ever since their inception in the late 1800s. More recently, the theories and techniques of antennas have been extended into the optical regime, allowing for applications in biological sensing and energy harvesting [1]. All of these applications can be greatly enhanced by introducing some degree of reconfigurability to the antenna design and operation. The most common way to achieve this behavior is through the use of loading schemes [2]. The main objective of loading is to control an antenna's current distribution in some form, and thus allow for a substantial modification of the antenna's radiation characteristics [2]. As a result, analytically determining the current distribution of a loaded antenna is paramount in achieving insight into the operation of the antenna. An especially promising design that has been considered is the thin wire-loop antenna [3]. A full derivation of the current on a nanoloop antenna valid from the RF to optical regimes was developed by McKinley in 2013 [3] and was later extended to include the effects of loading [4]. Once the current distribution of the loop has been found, the corresponding far-field pattern can be determined using expressions derived by Werner [5] and Lu et al. [6]. The fully analytical nature of the equations describing the current distribution and far-field radiation characteristics make the design of loaded loop antennas an ideal candidate for optimization. Utilizing these analytical expressions removes the requirement to perform much longer simulations based on full-wave solvers such as a method of moments tool. This increase in speed is even more pronounced when computing the currents and far-fields of plasmonic and dispersive materials in the optical regime (i.e., for metallic nanoloop antennas). In this paper, the covariance matrix adaptation evolutionary strategy (CMA-ES) [7,8] is employed in order to optimize the loading configuration of loop antennas, making a high degree of radiation pattern control possible.

\section{THEORETICAL FORMULATION}

The geometry of the antenna is designated by loop radius $b$ and wire radius $a$, as illustrated in Fig. 1. In order to satisfy the thin-wire approximation used in these derivations, the dimensions must satisfy the condition that $b \gg$ a. In this paper, the thickness factor, defined as $\Omega=2 \ln (2 \pi b / a)$, will be adopted [4]. Multiple voltage sources and/or impedance loads can be placed around the loop as shown in Fig. 1.

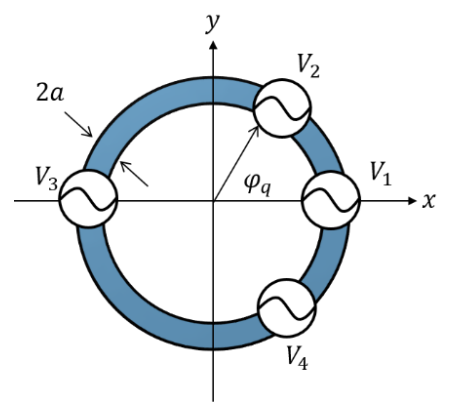

Fig. 1. Geometry of loaded loop with asymmetric load placement

Expressions for the currents and far-fields of a loaded loop antenna are reproduced below for reference:

$$
I(\varphi)=\sum_{q=1}^{M}\left[\sum_{m=-\infty}^{\infty} Y_{m}^{\prime} e^{j m\left(\varphi-\frac{2 \pi(q-1)}{M}\right)}\right] V_{q}
$$




$$
\begin{gathered}
E_{\theta}^{\prime}=-j \frac{\eta_{0} e^{-j k_{0} r} \cot \theta}{2 r} \sum_{m=1}^{\infty} m j^{m}\left\{I_{m} e^{j m \varphi}-I_{-m} e^{-j m \varphi}\right\} J_{m}(\mathrm{w}) \\
E_{\varphi}^{\prime}=-\frac{\eta_{0} e^{-j k_{0} r} k_{b}}{2 r} \sum_{m=0}^{\infty} \epsilon_{m} j^{m}\left\{I_{m} e^{j m \varphi}+I_{-m} e^{-j m \varphi}\right\} J_{m}^{\prime}(w)
\end{gathered}
$$

The derivation and description of these equations is further elaborated upon in [11-12].

\section{PEC CASE: MotivATING EXAMPLE}

Due to the fully analytical nature of the expressions in (1)(3), they may be implemented efficiently within a global optimizer using MATLAB [9]. In this first example, CMA-ES is employed to perform a single objective, four variable study of a PEC loop under a variety of different loading schemes. Physical insight about the dissipative nature of resistors inspired the original design choice of placing a resistor directly opposite the feed point to suppress radiation in the $(\theta, \phi)=$ $\left(90^{\circ}, 180^{\circ}\right)$ direction. This resulted in a mildly directive pattern. Symmetrically spaced capacitors were then added and shown to significantly enhance this directive behavior. This approach was then generalized further by allowing both the locations and impedances of loads to vary around the periphery of the loop in order to optimize the FBR, while maintaining the dissipative resistive load. The loads were modeled using the normalized unit-less parameters $r, l_{e}$, and $l_{u}$ which represent a given load's resistance, capacitance and inductance respectively [4]:

$$
Z_{L} \equiv \eta_{0}\left[r+j\left(k_{b} l_{\mu}-\frac{1}{k_{b} l_{\epsilon}}\right)\right]
$$

The resulting far-field pattern at $\theta=90^{\circ}$ can be seen in Fig. 2. These analytical results were validated using FEKO [10].

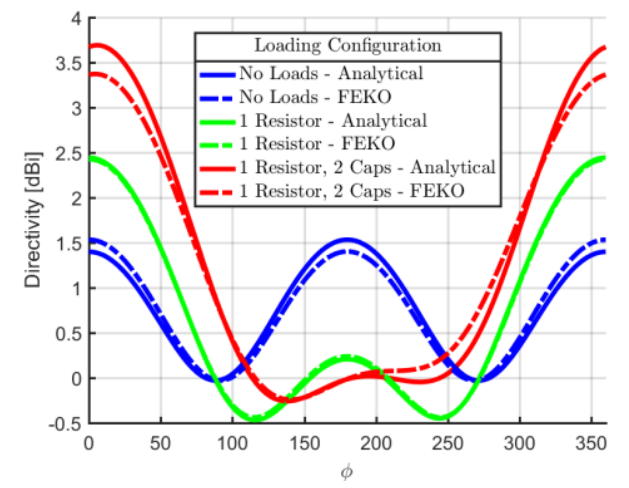

Fig. 2. Directivity of PEC unloaded and loaded loop with $k_{b}=0.344$, $\Omega=12, r=0.15$ at $\phi=180^{\circ}, l_{e}=0.110$ and 0.012 at $\phi=22.8^{\circ}$ and $338.5^{\circ}$, respectively.

As seen above, adding the capacitances increases the directivity in the forward direction by as much as $1 \mathrm{~dB}$, while maintaining a low directivity in the backward direction.

\section{LOADING APPLIED TO NANOLOOPS}

The expressions in (1)-(3) can easily be extended to the optical regime by including dispersive effects [3, 6]. As lumped impedances are not easily available in the optical regime, a first order capacitance model was used to represent the impedances of sections within the loop. This model specifies the area of the capacitor in terms of the angular width $d$ of the impedance section. As demonstrated in Fig. 3, adding capacitive loads to a nanoloop significantly modifies its directivity. Importantly, the energy directed broadside, as evidenced by the presence of a large null, has now been concentrated within the plane of the loop. This results in an enhanced directivity in the $\phi=0^{\circ}$ direction.

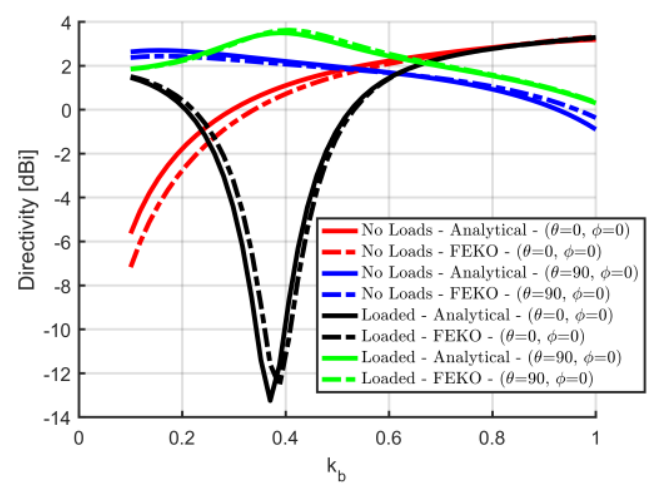

Fig. 3. Directivity of unloaded and loaded nanoloop with $b=477.5$ $\mathrm{nm}, \Omega=9.81, \epsilon_{r}=15$,_-angular width $d=5^{\circ}$ with capacitive loads located at $\phi=90^{\circ}$ and $270^{\circ}$.

\section{CONCLUSION}

The analytical development of loop antennas, both alone and with loading, has readily made available the potential to perform rapid parametric studies on loops ranging from the RF to the optical regimes. The two examples presented are merely a small sample of what is possible when this analytical theory is combined with the utility of global optimization.

\section{REFERENCES}

[1] C. A. Balanis, Antenna Theory. New York: Wiley, 1997.

[2] J. T. Bernhard, "Reconfigurable antennas," Synthesis Lectures on Antennas, vol. 2, no. 1, pp. 1-66, 2007.

[3] A. F. McKinley, T. P. White, and K. R. Catchpole, "Theory of the circular closed loop antenna in the terahertz, infrared, and optical regions," J. Appl. Phys., vol. 114, no. 4, p. 044317, 2013.

[4] A. F. McKinley, "Theory of impedance loaded loop antennas and nanorings from RF to optical wavelengths," IEEE Trans. Ant. and Prop., vol. 65 , no. 5, pp. 2276-2281, 2017.

[5] D. H. Werner, "An exact integration procedure for vector potentials of thin circular loop antennas," IEEE Trans. Ant. and Prop., vol. 44, pp. 157-165, 1996

[6] B. Q. Lu, J. Nagar, T. Yue, M. F. Pantoja, and D. H. Werner, "Closedform expressions for the radiation properties of nanoloops in the terahertz, infrared and optical regimes," IEEE Trans. Ant. and Prop., vol. 65, no. 1, pp. 121-133, 2017.

[7] N. Hansen, and A. Ostermeier, "Completely derandomized selfadaptation in evolutionary strategies," Evol. Comput., vol. 9, no. 2, pp. 196-195, 2001

[8] M. D. Gregory, Z. Bayraktar, and D. H. Werner, "Fast optimization of electromagnetic design problems using the covariance matrix adaptation evolutionary strategy," IEEE Transactions on Antennas and Propagation, vol. 59, no. 4, pp. 1275-1285, April 2011.

[9] MATLAB R2014A, Mathworks - Natick, MA, USA, https://www.mathworks.com/

[10] FEKO Suite 7.0, EM Software \& Systems - S.A., Stellenbosch, South Africa, http://www.feko.info 